\title{
Increasing the Refractive Index of a Germanosilicate Planar Waveguide by Irradiation with an Infrared Ultrashort Pulse Laser
}

\author{
Hikaru KOUTA, Yutaka URINO*, Tadahiko HANADA*, and Kazuyuki HIRAO** \\ Functional Materials Research Laboratories, NEC, Kawasaki 216-8555 \\ *Fiber Optic Devices Division, NEC Networks, Maki 747, Ohtsuki-cho, Ohtsuki 401-0016 \\ **Faculty of Engineering, Kyoto University, Yoshida-honcho, Sakyo, Kyoto 606-8501
}

(Received December 17, 2001)

\begin{abstract}
Reported here is the use of the focused beam of an infrared ultrashort pulse laser ( 150 femtoseconds pulse width, $200 \mathrm{kHz}$ repetition rate, $800 \mathrm{~nm}$ wavelength, and $100 \mu \mathrm{m} / \mathrm{s}$ scanning speed) to increase the refractive index of a germanium-doped silica-glass planar waveguide. The increase obtained, $\Delta n$, itself increases in proportion to increases in the laser intensity of the surface irradiation, and it saturates to $1.6 \sim 1.8 \times 10^{-3}$ at 2.2 $\mathrm{TW} / \mathrm{cm}^{2}$, without producing any increase in insertion loss and birefringence. Further, no decay in the refractive index change is observed after annealing at $200{ }^{\circ} \mathrm{C}$ for 10 hours. Results show that ultrashort pulse lasers are more suitable than UV lasers as light sources for highly stable adjustment of the optical lengths of waveguide devices.
\end{abstract}

Key Words: Ultrashort pulse laser, Refractive index change, Optical length adjustment, Planar lightwave circuit, Germanium-doped silica glass waveguide

\section{Introduction}

For use in Dense Wavelength Division Multiplexing (DWDM) photonic networks, optical waveguide devices require ever more precisely adjusted device characteristics, and this means greater demand for homogeneity in the refractive indices of such devices. Ordinary process deviations result in insufficient homogeneity, however, and subsequent adjustment of refractive indices becomes necessary.

Because irradiation with UV light increases the refractive index of a Ge-doped silica-glass waveguide, UV lasers have been used to adjust optical waveguide characteristics. ${ }^{1)}$ It has recently been reported that irradiation by a focused infrared ultrashort pulse laser beam can, because of the multi-photon absorption effect, create stable increases in the refractive indices of various types of glass, ${ }^{2-4)}$ which suggests that infrared ultrashort pulse lasers might be used as light sources for adjusting optical waveguide characteristics. In this paper we report just such a use, with particular attention to the questions of insertion loss and of the thermal stability of the refractive index after irradiation. We consider here in the use of ultrashort pulse lasers in comparison to that of UV lasers, with particular respect to its potential for application to the adjustment of device characteristics.

\section{Method for Measuring Changes in Refractive Indices of Waveguides}

In order to measure changes in the refractive indices of waveguides, we first designed a planar lightwave circuit (PLC) to serve as a Mach-Zehnder (MZ) interferometer (see Fig. 1). The PLC (width $32 \mathrm{~mm}$ ) has two ports and a $3 \mathrm{~dB}$ directional coupler on each end. The input-side coupler divides a signal, and the resulting two signals pass through separate arms, the difference in optical length of their respective paths resulting in a phase difference that produces interference in the out-put side coupler.

Figure 1 shows the setup for determining the interference spectra. The amplified spontaneous emission (ASE) light from an Er-doped fiber serves as a broadband light source (approx. 1.55 $\mu \mathrm{m})$, and the input light is polarized. The transmission spectra of the output light are determined by an optical spectrum analyzer (OSA) at a resolution of $0.05 \mathrm{~nm}$. The spectra are normalized by subtracting a reference spectrum obtained directly from the ASE source.

In our experiments, we subjected the shorter of the two arms to laser beam irradiation, which resulted in a change in optical length and, consequently, in interference wavelength. We calculated the value of the change in the refractive index $(\Delta n)$ as

$$
\Delta n=(\lambda \cdot \Delta \lambda) /(F S R \cdot \Delta l)
$$

where FSR (free spectral range) is set to $16.2 \mathrm{~nm}$ and may be expressed as $\lambda / m$ ( $m$ : order of diffraction), $\Delta \lambda$ is the shift in interference wavelength, and $\Delta l$ is the scanning length of the laser

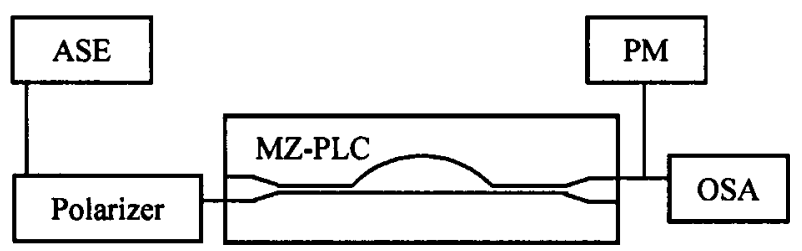

Fig. 1 Setup for measurement of interference spectra in MZPLC device. 
beam.

\section{Sample Preparation and Beam Irradiation}

The PLC was fabricated on a Si substrate using atmospheric pressure chemical vapor deposition (APCVD) and reactive ion etching (RIE) ${ }^{5)}$ A Ge-doped core ( $7 \%$ concentration; $5.5 \times$ $5.5 \mu \mathrm{m})$ was created. The difference in relative refractive indices between the core and the cladding was $0.7 \%$. An MZ interferometer was patterned for use in $\Delta n$ measurements, and a separate straight wave guide was made for use in insertion loss measurements.

The experimental setup for the irradiation is shown in Fig. 2. The laser pulses used to induce change in the refractive index were obtained from a regeneratively amplified $\mathrm{Ti}^{3+}: \mathrm{Al}_{2} \mathrm{O}_{3}$ laser pumped by an Ar ion laser. The pulse width was 150 femtoseconds (fs), the wavelength was $800 \mathrm{~nm}$, the repetition rate was $200 \mathrm{kHz}$, and the maximum average power was 800 $\mathrm{mW}$. The laser beam was focused through a microscope (using both $10 \times$ and $20 \times$ objective lenses). The device was observed through a CCD camera mounted over the microscope to watch for any visible physical changes that might be produced during the irradiation. The PLC, which was fixed on a computer-controlled XYZ stage, was irradiated along the shorter arm of its interferometer. The scanning length was kept to $2 \mathrm{~mm}$ by coordinating the scanning speed with the open-time of a mechanical shutter. The speed that we used, $100 \mu \mathrm{m} / \mathrm{s}$, is one which had previously been reported to work well in the direct writing of waveguides, with $120 \mathrm{fs}$ laser pulses at a $200 \mathrm{kHz}$ repetition rate. ${ }^{3,4)}$ The pulse energy was controlled by means of a neutraldensity (ND) filter. In order to induce a homogeneous interaction in the focus area, a laser beam $3 \mathrm{~mm}$ in diameter (with a Gussian-like profile) was first expanded to $10 \mathrm{~mm}$ and then sent through a $5 \mathrm{~mm}$ aperture (top-hat-like profile) before being focused through the objective lens.

\section{Results}

The surface area above the core has a cylindrical mound shape. Since a circular beam spot will become elliptical on the surface of a sample of this shape, neither the exact area of the beam spot nor the level of the of input energy can easily be calculated with precision, and that is why we chose to use intensity $\left(\mathrm{W} / \mathrm{cm}^{2}\right)$ of surface irradiation, rather than that of spot area in the waveguide, as our beam parameter. Intensity was set by adjusting both pulse energy and the surface irradiation spot size (to approx. $16 \mu \mathrm{m}$ in

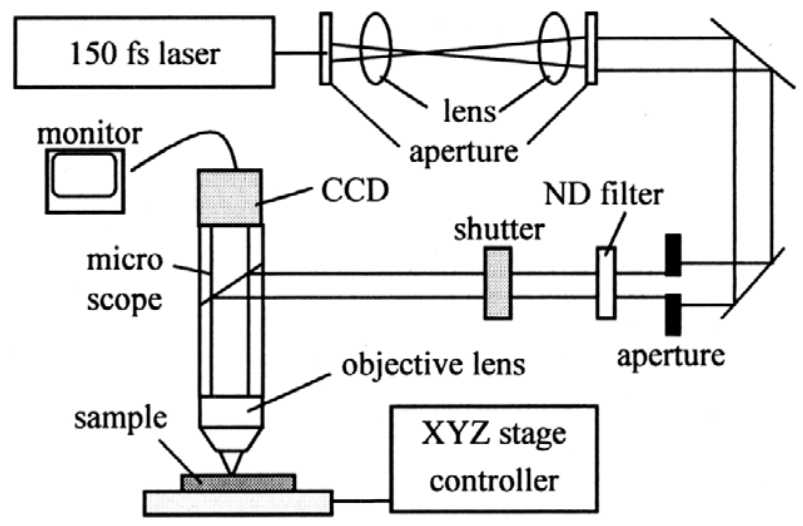

Fig. 2 System for laser irradiation by direct beam scanning. diameter).

Figure 3 shows transmission spectra before and after irradiation at $1.08 \mathrm{TW} / \mathrm{cm}^{2}(65 \mathrm{~mW}$ average power). The interference wavelengths (two bottom peaks) show a shift of $5.20 \mathrm{~nm}(\Delta \lambda)$. We then used Equation 1 to calculate $\Delta n$ values on the basis of the obtained $\Delta \lambda$.

The change in refractive index of a Ge-doped waveguide is shown in Fig. 4. With a $10 \times$ lens, $\Delta n$ jumped up to $0.7 \times 10^{-3}$ (at $\left.1.1 \mathrm{TW} / \mathrm{cm}^{2}\right)$ and then gradually increased to $1.2 \times 10^{-3}$ at $(5.4$ $\left.\mathrm{TW} / \mathrm{cm}^{2}\right)$ (Fig.4(a)). With a $20 \times$ lens, $\Delta n$ quickly increased to $1.6 \times 10^{-3}$ (at $2.2 \mathrm{TW} / \mathrm{cm}^{2}$ ) and then, at a reduced rate of increase, to a saturated $\Delta n$ value of $1.8 \times 10^{-3}\left(5.4 \mathrm{TW} / \mathrm{cm}^{2}\right)$ (Fig.4 (b)). When intensity was increased to above $5.4 \mathrm{TW} / \mathrm{cm}^{2}$, a dark damage-line was observed along the core-clad interface. The difference in $\Delta n$ between the TE and TM modes seen in Fig. 4 (b) is less than $2 \%$ when the intensity is higher than $4.3 \mathrm{TW} /$ $\mathrm{cm}^{2}$.

Changes in insertion loss resulting from laser irradiation were determined in a set-up that included a straight waveguide, a 1.55 $\mu \mathrm{m}$ diode laser for a light source, and a power meter (PM). If the refractive index of the clad around the core were to be increased by irradiation, insertion loss would also increase because of increased scattering loss at the core/cladding interface. As Fig. 5 shows, however, the deviations in insertion losses of TE and TM modes over the range of intensity values tested (with a $20 \times$ lens focus) are negligible ( $\pm 0.1 \mathrm{~dB}$; within experimental error).

Results also show that no damage due to insertion loss occurred in the waveguide for the measured power range (we measured up to $3.3 \mathrm{TW} / \mathrm{cm}^{2}$ ). The increase in refractive index appears to have occurred, then, entirely within the core area alone.

Figure 6 shows the effect of annealing (at $200{ }^{\circ} \mathrm{C}$ for 10 hours) on the increased refractive indices produced in the irradiation that is shown in Fig. 4 (b). The results shown here are sufficient to confirm the long-term reliability of DWDM optical devices produced in the manner described. While the increased refractive index produced at $1.1 \mathrm{TW} / \mathrm{cm}^{2}$ decreased by $10-20 \%$, indices produced at $2.2 \mathrm{TW} / \mathrm{cm}^{2}$ and above showed negligible decay, indicating that saturated $\Delta n$ values offer high stability with respect to heat.

\section{Discussion}

With respect to UV laser irradiation, two processes have been

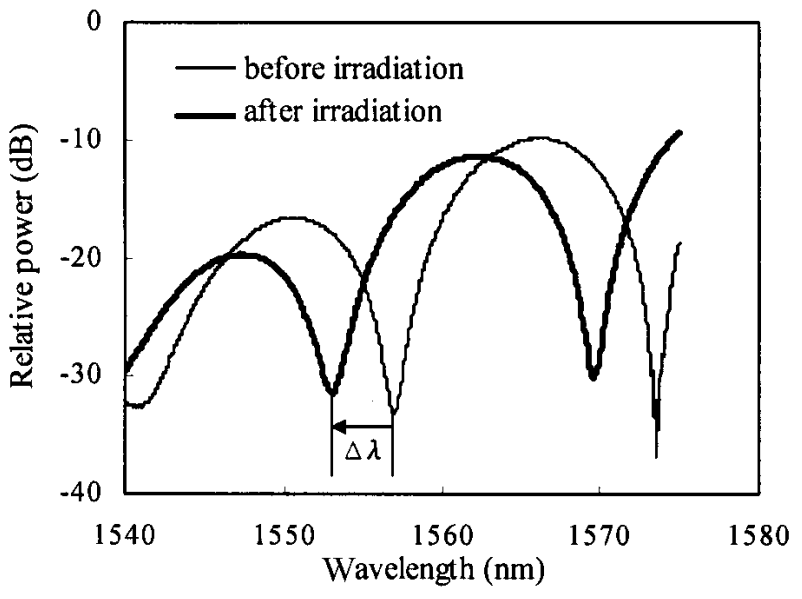

Fig. 3 Change of transmission spectra in MZ-PLC with ultrashort pulse laser irradiation. 

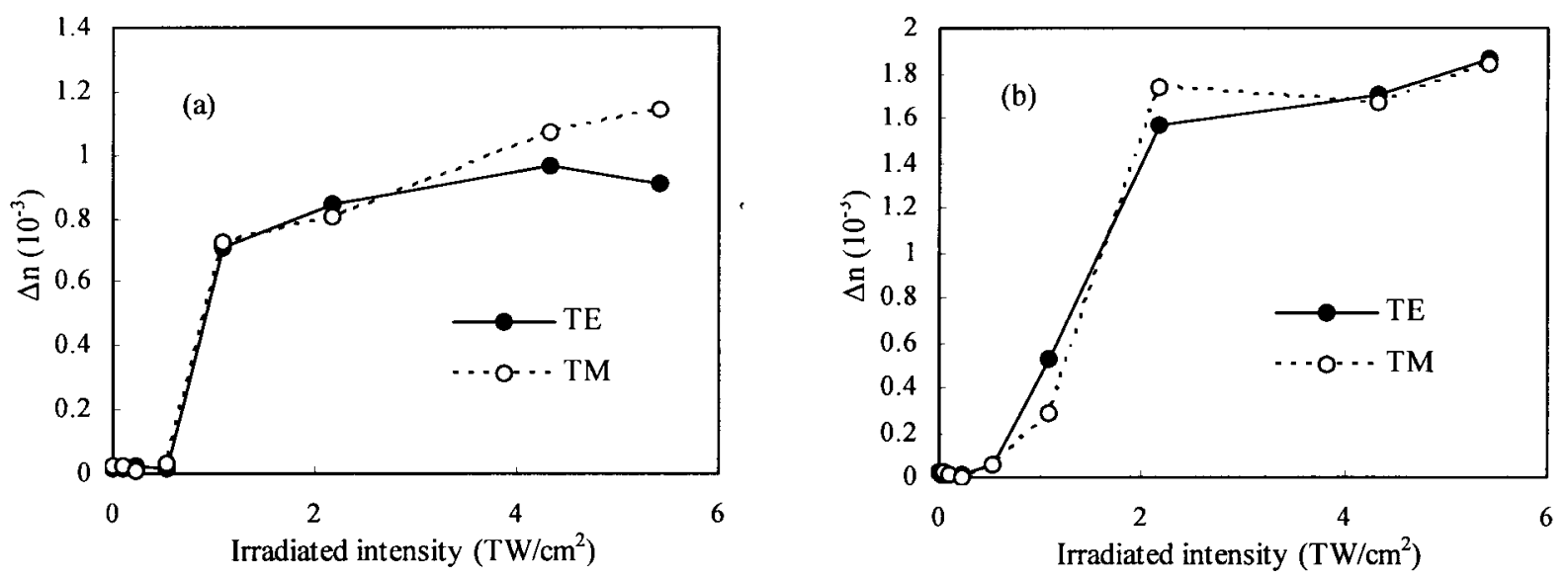

Fig. 4 Increases in refractive indices of Ge-doped wave-guide with ultrashort pulse laser, 150 femtoseconds pulse, $100 \mu \mathrm{m} / \mathrm{s}$ scanning speed, and $2 \mathrm{~mm}$ long scanning length; (a) 10× objective lens, (b) 20x objective lens.

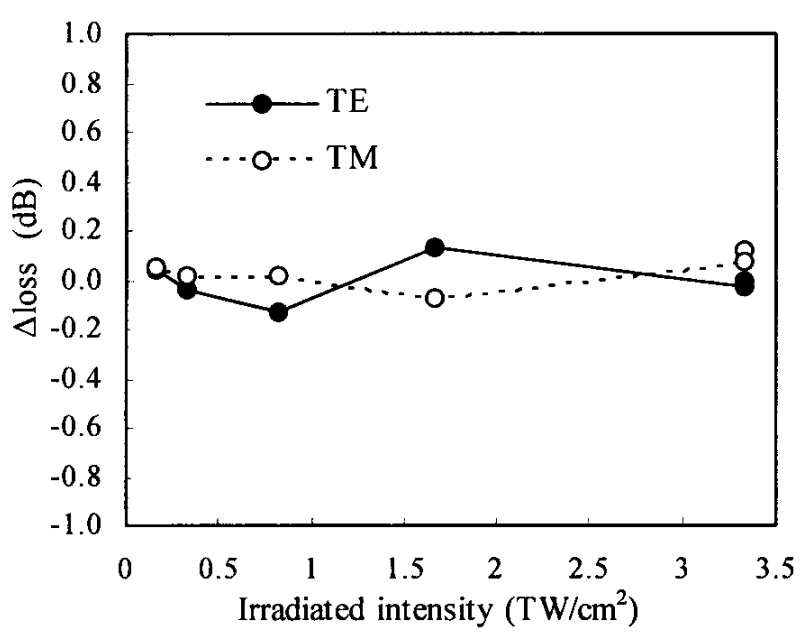

Fig. 5 Change of insertion loss vs. irradiated intensity.

suggested as explaining the increased refractive indices in $\mathrm{Ge}$ doped silica glass: (1) the formation of Ge-related defects, such as GeE' centers, due to photochemical reactions, ${ }^{6}$ ) and (2) the densification of the matrix around Ge-atoms due to thermally induced structural relaxations. ${ }^{7)}$ In fact, the increase would appear to be the result of a complex interaction of the two. When Ge-doped glass is irradiated with low-fluence UV light, $\Delta n$ reaches an order of $10^{-4},{ }^{8,9)}$ and with high-fluence UV light, an order of $10^{-3}$, saturating at $1 \sim 2 \times 10^{-3},{ }^{10-12)}$ and enhancements achieved through hydrogen loading only contribute to the reaching of a saturated $\Delta n$ of $3.4 \times 10^{-3} .13$ )

With respect to $\Delta n$ achieved with focused infrared ultrashort pulse laser irradiation, a previous report attributes a $2 \times 10^{-2}$ level densification of silica glass that is unrelated to any effect on Ge ions. ${ }^{2,14,15)}$ In our study, by way of contrast, in which the increase is of the order of $10^{-3}$ (saturation at $1.6 \sim 1.8 \times 10^{-3}$ ), the index increase is limited to the Ge-doped core alone, indicating that it is due, rather, to the same complex combinations of Gerelated mechanisms previously noted for UV-laser irradiation. If the surface of the waveguide were flat, the refractive index of the Ge-undoped clad would also be changed, along with that of the core, by infrared ultrashort pulse laser irradiation whose intensity is greater than $1 \mathrm{TW} / \mathrm{cm}^{2}{ }^{2,14)}$ In our study, however, the surface area above the core has a cylindrical mound shape. The intensity at the spot in the waveguide decreases to the order of
$\mathrm{GW} / \mathrm{cm}^{2}$ because the reflection ratio at the surface is higher than would be the case with a flat surface, and the beam would has the same effect as that of a UV laser.

With infrared ultrashort pulse laser irradiation, the increase in refractive index results from multi-photon absorption, which means that the increase will occur only in the area upon which the irradiation is focused, ${ }^{16-18)}$ and the index of the core can be changed without creating the excessive absorption loss in the clad layer covering the core. With UV irradiation, on the other hand, the increase results from two-photon absorption, which means that the UV light is easily absorbed in the clad layer, and the resulting penetration is shallow. ${ }^{10)}$

Though the exact control of ultrashort pulse intensity at the core is difficult, we can irradiate the core with sufficient power to ensure a saturated $\Delta n$ despite any possible small anomalies in the physical processes. In order to use the achievable increase in refractive index to adjust the optical length of a waveguide, we control the beam-scanning-length parameter. We can, for one example, produce an optical length in the range of $2 \pi(1.55$ $\mu \mathrm{m}$ ) with a scan-length of $0.97 \mathrm{~mm}$ (for a saturated $\Delta n$ of $1.6 \times$ $\left.10^{-3}\right)$.

As for the robustness with respect to annealing of our saturated $\Delta n$ values (Fig. 6), it would appear that any Ge-related, thermally unstable centers initially present in the core had already been eliminated during the fs-laser beam irradiation, by

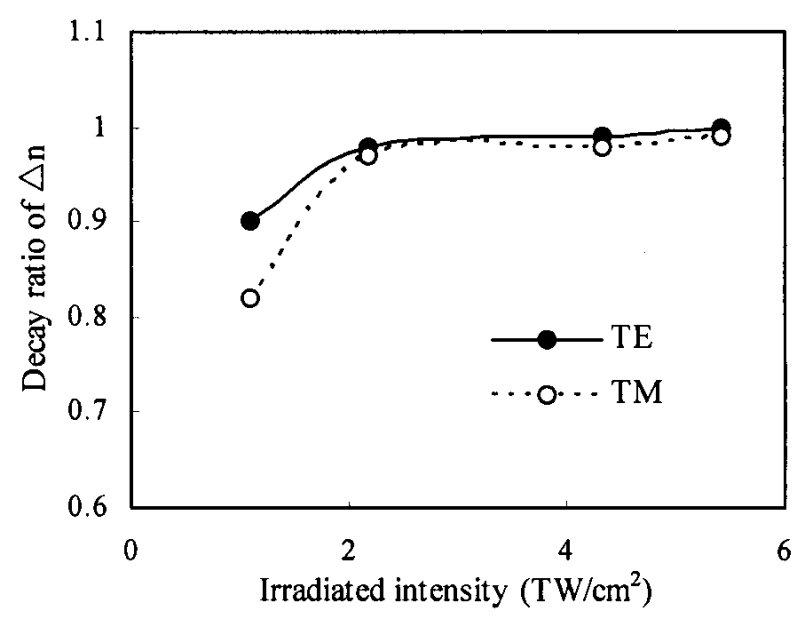

Fig. 6 Thermal decay ratio vs. irradiated intensity. 
the heat created by the laser energy applied in the irradiation area, and no centers were later there to create decay in refractive index values. This is in marked contrast to the thermal instability of UV-induced increases, which will occur even at temperatures below $100{ }^{\circ} \mathrm{C}$ due to the effect of defects related to the structural relaxation of GeE' centers. ${ }^{11,19)}$ This difference represents a very significant advantage for ultrashort pulse laser irradiation over that of UV laser radiation for the adjustment of the characteristics of optical devices. Further, with our method, we are able to measure optical device characteristics during laser irradiation itself, and use the data as feedback for any needed adjustments.

\section{Summary}

The refractive index of a germanium-doped silica-glass planar waveguide has been increased to a saturated level of $1.6 \sim$ $1.8 \times 10^{-3}$ with infrared ultrashort pulse laser irradiation. Insertion loss in waveguides showed no increase due to this irradiation, and annealing at $200{ }^{\circ} \mathrm{C}$ for 10 hours produced no decay in the refractive index achieved for this saturated value. The results here show ultrashort pulse lasers to be superior to UV lasers as light sources for adjusting optical length.

\section{Acknowledgement}

The authors wish to expression their appreciation for the valuable advice offered by K. Miura and S. Fujiwara of Central Glass Co., Ltd.

\section{References}

1) D. A. Zauner, J. Hubner, K. J. Malone, and M. Kristensen: Electron. Lett. 34 (1998) 780.

2) K. M. Davis, K. Miura, N. Sugimoto, and K. Hirao: Opt. Lett. 21 (1996) 1729

3) K. Miura, J. Qiu, H. Inoue, T. Mitsuyu, and K. Hirao: Appl. Phys. Lett. 71 (1997) 3329.

4) Y. Kondo, K. Nouchi, T. Mitsuyu, M. Watanabe, P. G. Kazansky, and K. Hirao: Opt. Lett. 24 (1999) 646.

5) T. Hanada, T. Shimoda, M. Kitamura, and S. Nakamura: IEICE Trans. Electron. E80-C (1997) 130.

6) H. Hosono, Y. Abe, D. L. Kinser, R. A. Weeks, K. Muta, and H. Kawazoe: Phys. Rev. B 46 (1992) 11445.

7) J. Nishii, K. Fukumi, H. Yamanaka, K. Kawamura, H. Hosono, and H. Kawazoe: Phys. Rev. B 52 (1995) 1661.

8) C. V. Poulsen, J. Hubner, T. Rasmussen, L. -U. A. Anderson, and M. Kristensen: Electron. Lett. 31 (1995) 1437.

9) J. B. Jensen, P. Varming, B. Liu, and W. Gries: OFC 2001 WDD901 (2001).

10) K. P. Chen, P. R. Herman, and J. Zhang: OFC 2001 WDD81-1 (2000).

11) J. Albert, B. Malo, K. O. Hill, F. Bilodeau, D. C. Johnson, and S. Theriault: Appl. Phys. Lett. 67 (1995) 3529.

12) Y. Urino, N. Ofusa, T. Saito, T. Shimoda, and T. Hanada: CLEO Pacific Rim FH1 (1999) 1141

13) P. J. Lemaire, R. M. Atkins, V. Mizrahi, and W. A. Reed: Electron. Lett. 29 (1993) 1191.

14) S. -H. Cho, H. Kumagai, M. Obara, and K. Midorikawa: Opt. Commun. 168 (1999) 287.

15) K. Yamada, W. Watanabe, T. Toma, and K. Itoh: Opt. Lett. 26 (2001) 19.

16) J. Qiu, K. Miura, and K. Hirao: Jpn. J. Appl. Phys. 37 (1998) 2263.

17) M. Watanabe, H. -B. Sun, S. Juodkazis, T. Takahashi, S. Matsuo, Y. Suzuki, J. Nishii, and H. Misawa: Jpn. J. Appl. Phys. 37 (1998) 1528.

18) S. Kawata, H -B. Sun, T. Tanaka, and K. Tanaka: Nature 412 (2001) 697.

19) S. R. Baker, H. N. Rourke, V. Baker, and D. Goodchild: J. Lightwave Technol. 15 (1997) 1470 